\title{
Accuracy of Multilevel Registration in Image-guided Pedicle Screw Insertion for Adolescent Idiopathic Scoliosis
}

Jun Takahashi, MD, Hiroki Hirabayashi, MD, Hiroyuki Hashidate, MD, Nobuhide Ogihara, MD, Hiroyuki Kato, MD.

Department of Orthopedic Surgery, Shinshu University, School of Medicine,

3-1-1 Asahi, Matsumoto, Nagano, 390-8621, Japan

Phone: 81-263-37-2659

FAX: 81-263-35-8844

e-mail: jtaka@shinshu-u.ac.jp

Please address all reprints and correspondence to:

Jun Takahashi, MD

Department of Orthopaedic Surgery, Shinshu University, School of Medicine 3-1-1 Asahi, Matsumoto, Nagano, 390-8621, Japan

Phone: $81-263-37-2659$

Fax: $\quad 81-263-35-8844$

Email: jtaka@ shinshu-u.ac.jp

Running Head: Accuracy of Multilevel Registration 


\section{MINI ABSTRACT}

Accuracy of multilevel registration for skip pedicle screw placement during image-guided, computer-assisted spine surgery, in adolescent idiopathic scoliosis was assessed. Mean surgical time: $310 \mathrm{~min}$. Pedicle violation observed in only 4/265 screws (1.5\%). Mean registration error after point merge: $1.69 \pm 0.52$ : after surface merge $0.51 \pm 0.16$. Multilevel registration may decrease operative time without compromising accuracy of pedicle screw placement. 


\section{KEY POINTS}

- Multilevel registration employs more than 6 registration points for two, three or four consecutive vertebra to shorten the surgical time.

- Pedicle violation was observed in $1.5 \%$ of inserted screws.

- Multilevel registration may decrease operative time without compromising accuracy of pedicle screw placement afforded by this technique in the setting of adolescent idiopathic scoliosis.

- Mean correction rate of skip pedicle screw instrumentation was $66.2 \%$. 


\section{STRUCTURED ABSTRACT}

Study Design: Retrospective clinical study.

Objective: To assess the accuracy of multilevel registration for skip pedicle screw placement during image-guided, computer-assisted spine surgery, in the setting of adolescent idiopathic scoliosis (AIS).

Summary of background data: Computerized frameless stereotactic image-guidance has been used recently to improve pedicle screw placement accurately and safety during spine surgery. Because of possible intervertebral motion and usual difference in patients' position between preoperative imaging and surgery, the imaging model and the surgically exposed spine may be significantly discordant. Consequently, current protocols suggested separate registration of each spinal level (single-level registration) before respective pedicle screw placement, a timeconsuming process. Moreover, although multilevel registration for lumbar spine has been reported, that for thoracic spine has not.

Methods: Nineteen patients ( 1 male, and 18 females; mean age, 13.9 years) with AIS who underwent multilevel registration for skip pedicle screw placement were included. Variables including surgical time, blood loss, preoperative and 2-year post-operative Cobb angle, correction rate, and post-operative screw position by CT image were evaluated. Mean registration error (MRE) after point merge and again after surface merge were recorded for each consecutive vertebra of each case.

Results: Mean surgical time was $310 \mathrm{~min}$. (range, 168-420 min.). Mean blood loss $1138 \mathrm{~g}$ (range, 300-2300 g). Cobb angle before operation and at 2 years post-operation was 62.4 (43100) degrees and 21.6 (9-42) degrees, respectively. Mean correction rate $66.2 \%$ (39.7-84.5\%). Total 265 screws were inserted with CT-based navigation system. Pedicle violation was observed in only 4 screws (1.5\%). No neurovascular complication occurred. After point merge, average MRE of all cases was $1.69 \pm 0.52 \mathrm{~mm}$, and after surface merge was $0.51 \pm 0.16 \mathrm{~mm}$.

Conclusions: Multilevel registration may decrease operative time without compromising accuracy of pedicle screw placement afforded by this technique in the setting of AIS.

Key Words: computer-assisted surgery, pedicle screw, registration, multilevel, adolescent idiopathic scoliosis, spinal navigation 


\section{INTRODUCTION}

The application of a spinal navigation system to surgery represents a major technological advance. Computer-assisted implantation of pedicle screws has been primarily limited to the lumbar spine ${ }^{1-3}$, and recently the efficiency of screws for thoracic and cervical pedicles has been reported $^{4-8}$. There are several methods for computer-assisted pedicle screw placement including frameless stereotaxy ${ }^{9}$ and fluoroscopic navigation ${ }^{10}$, while the clinical application of 3dimensional computer-assisted surgery has significantly reduced the misplacement rate of screws over the last decade ${ }^{1,11-13}$. Clinical studies demonstrated an improved accuracy in pedicle screw insertion using a computed tomography (CT)-based navigation system, which eliminates exposure to fluoroscopic radiation ${ }^{14}$.

Pedicle screws have been recognized as a powerful option for deformity constructs. Segmental pedicle screw instrumentation in adolescent idiopathic scoliosis (AIS) enables a shorter fusion length and a better correction ${ }^{15,16}$. However, both neurovascular and visceral structures are at potential risk from screw misplacement owing to pedicle drift caused by significant deformity ${ }^{17-20}$. Liljenqvist et $a l^{21}$ demonstrated that pedicle screw instrumentation on the concavity in the apical region is critical because of the small endosteal pedicle width, which precludes safe screw placement in that area.

During preoperative data acquisition scans, patients are most often supine; however, during surgery they are most often prone. Intervertebral motion resulting from this may result in a significant discordance between the imaging model of the spine and the surgically exposed spine $^{22}$. Consequently, current manufacturer protocols recommend registering each spinal level (single-level registration) separately before pedicle screw placement at that level. This is intended to increase the accuracy of the registration process but may be associated with an increased operative time. Although the accuracy of multilevel registration for multilevel pedicle screw placement during image-guided, computer-assisted lumbar spine surgery has been reported $^{23}$, that for thoracic spine has not. Use of multilevel registration can potentially decrease operative time. The purpose of this study was to assess the accuracy of multilevel registration during computer- assisted, image-guided spinal surgery, in the setting of AIS.

\section{MATERIALS AND METHODS}

This study reviewed 19 consecutive patients with AIS who underwent correction with posterior skip pedicle screw instrumentation performed by a single spinal surgeon (J.T.), with minimum 2 -years follow-up. The mean age at the time of surgery was 13.9 (11 to 17) years, and the male-to-female ratio was 1 to 18 . Nine curves were classified as type 1 , five as type 2 , and five as type 3 , according to the system Lenke, et $a l^{24}$. The mean preoperative magnitude of the major curve measured by the Cobb angle was 62.4 \pm 13.9 (mean \pm standard deviation) (range, 43100 ) degrees. The mean preoperative kyphotic angle from T5 to T12 was $6.6 \pm 10.2$ (range, -1435 ) degrees. Preoperative apical vertebral rotation of main curve using CT ${ }^{25,26}$ was $19.8 \pm 8.1$ (range, 7-36) degrees. A total of 265 pedicle screws were placed using the multilevel registration. Intraoperative neuromonitoring using motor evoked potentials was performed in all patients. Preoperative, 1-week postoperative and 2-year postoperative radiographic parameters and clinical data was examined. Mean registration error (MRE) after point merge and again after surface merge were recorded for each consecutive vertebra of each case. Patient satisfaction at 
Accuracy of Multilevel Registration

2-year postoperatively was investigated through question 21 of SRS-22r Patients Questionnaire, "Are you satisfied with the results of your back management?"

\section{Navigation and surgical procedure}

A frameless stereotactic image-guidance system (Stealth Station TREON ${ }^{\mathrm{TM}}$; Medtronic, Sofamor Danek, Memphis, TN, USA) was used for planning the pedicle screw diameter and length as well as correct screw placement and fixation of the thoracic and lumbar spine. This group's procedure employs more than 6 registration points for two, three or four consecutive vertebra, to shorten the surgical time (multilevel registration) (Fig. 1). More accurate positioning is possible through use of the top of the spinous process and bilateral transverse process tip as points. Pedicle screw insertion to the upper and lower end was performed bilaterally, and insertion to the other points was basically performed with zigzag fashion. Pedicles with outer diameter of $4.5 \mathrm{~mm}$ or less were skipped by evaluation during navigation plan. If an upper end was skipped, a hook was used, and if three or more pedicle at other levels were skipped, sublaminar wiring with ultra-pliable polyethylene cable (Nespron cable system; Alfresa Pharma, Co. Osaka, Japan) was performed (Fig. 2, 3).

\section{Postoperative assessment of screw placement}

Screw position was evaluated one week after operation using an axial CT scan at each screw axis. Following the scheme of Rao et $a l^{27}$, the evaluation of screw malposition was classified as grade 0 (no apparent violation of the pedicle), grade $1(<2 \mathrm{~mm}$ perforation of the pedicle, with 1 screw thread out of the pedicle), grade 2 (between 2 and $4 \mathrm{~mm}$ of perforation of the pedicle, with half of the diameter of the screw outside of the pedicle), grade 3 (>4 mm or complete perforation of the pedicle), with grades 2 and 3 representing "violation." On the basis of this grading system, screw misplacement rates were determined. The medial or lateral perforation of the pedicle wall was also evaluated.

\section{RESULTS}

The time required to insert all screws using multilevel registration was $79.2 \pm 17.5$ (range, 55-118) minutes. Total surgical time was 310.1 \pm 79.1 (range, 168-420) minutes. Total blood loss was $1137.9 \pm 51.3$ (range, $300-2300$ ) $\mathrm{mL}$. The average number of instrumented spinal segments was 11.2 \pm 1.7 (range, 8-14 ) vertebra. The mean 1-week and 2-year postoperative magnitude of the major curve measured by the Cobb angle was 20.1 \pm 9.7 (range, 8-40) degrees and 21.6 \pm 10.5 (range, 9-42) degrees, respectively. Correction rate was 66.8 \pm 10.2 (range, 51.484.9 ) $\%$ and $66.2 \pm 11.9$ (range, $39.7 \pm 84.5$ ) \%, respectively. The mean 1-week and 2-year postoperative kyphotic angle from T5 to T12 was $12.9 \pm 5.4$ (range, 2-22) degrees and 13.5 \pm 7.1 (range, 4-30) degrees, respectively. The mean 1-week and 2-year postoperative apical vertebral rotation of main curve was 16.5 \pm 7.4 (range, 3-36) degrees and 17.6 \pm 7.5 (range, 5-36) degrees, respectively. After point merge, the average MRE of all cases was $1.69 \pm 0.52$ (range, 0.7-2.8) $\mathrm{mm}$. The mean MRE of all cases after surface merge was $0.51 \pm 0.16$ (range, 0.3-0.9) $\mathrm{mm}$. Of the 265 pedicle screws, $244(92.1 \%)$ were categorized as grade $0,17(6.4 \%)$ were grade $1,3(1.1 \%)$ were grade 2 , and $1(0.4 \%)$ was grade 3 (Fig. 4$)$. Only one grade 3 screw was seen, in a right T5 pedicle. Pedicle violation was observed in $1.5 \%$ of inserted screws. Pedicle violation in the lumbar, thoracolumbar, and thoracic spine was observed in $1.8 \%(1 / 56), 0.9 \%(1 / 115)$, and $1.4 \%$ 
(3/209) of inserted screws, respectively. No intraoperative complications such as neurovascular injury or adverse clinical consequences occurred as a result of pedicle perforation. No wound infection was observed. Patient satisfaction at 2-year postoperatively by SRS-22r Patients Questionnaire resulted in 10 very satisfied cases and 9 satisfied cases.

\section{DISCUSSION}

Vertebrae rotated toward the convex side that increase the angle of convergence of the pedicles and narrow pedicle width make it difficult to insert screws safely in the scoliotic rotated spine. There is a paucity of reports in the literature on the accuracy of pedicle screw placement in scoliosis surgery. In a report by Suk et al $^{28}$, the perforation rate of pedicle screw inserted by free hand in AIS posterior fusion was 1.5\%; however, only $10 \%$ of cases were evaluated by CT, and thus this reported rate is not highly reliable. In report by Kim and Lenke et $a^{29}$, the perforation rate by free hand technique was $7.9 \%$. The reported perforation rate by Halm et al ${ }^{16}$ was as high as $18.3 \%$ and the rate by Lilijenqvist et $a^{18}$ who employed fluoroscopy, was also as high as $25 \%$. In contrast, the perforation rates of reports of surgeries that employed navigation system ${ }^{30-32}$ were between 1.8 and $11.4 \%$. In the present report, the rate was as low as $1.5 \%$. The perforation rate of our multilevel registration was comparable to that reported by Kotani et al ${ }^{31}$, where in the perforation rate was $1.8 \%$ in single-level registration. In the evaluation of screw placement, more than $2 \mathrm{~mm}$ of penetration was defined as pedicle violation, for the reason that up to a $2 \mathrm{~mm}$ breach was considered a well-positioned screw since there are slight scatter effects seen on CT scans, even for titanium implants ${ }^{33}$.

Use of three-dimensional, image-guided technology requires that anatomic landmarks revealed during routine surgical exposure be matched to corresponding anatomic landmarks (fiducials) selected on a three-dimensional model of the spine created by a computer system using pre-acquired images, a process known as point merge. After the point merge process, the workstation reports the discordance between the exposed anatomic landmarks and the threedimensional computer model as an MRE. The MRE is not the actual error that will be observed clinically but a projected error estimated by the computer. The surgeon then identifies multiple bony points on the exposed spine, allowing the workstation to create a three-dimensional map of the exposed spine, a process known as surface merge. Utilization of a greater number of bony points for anatomic correlation is intended to further improve accuracy of the navigation systems (ie, a more accurate three-dimensional volumetric representation of the vertebral levels). The computer workstation again reports the discordance between the demarcated spinal anatomy and the three-dimensional computer model as an MRE. The complete process, involving point merge and surface merge, is known as registration. The burden of registration resulted in prolonged surgical time, thus the value of the procedure from the standardpoint of invasiveness was unclear. Papadopoulos et al $^{23}$ reported that the mean MRE after point merge by single-time multilevel registration in degenerative disorders of the lumbar spine was $1.78 \mathrm{~mm}$ and the average MRE after the surface merge was $0.70 \mathrm{~mm}$. In the AIS patients in our report, mean MRE by multilevel registration was as low as $1.69 \mathrm{~mm}$ and $0.51 \mathrm{~mm}$, and therefore these results show feasibility of this technique for the AIS patient.

This protocol with two sets of CT imaging was accepted by the study team to ensure the safety of surgery. Entrance surface dose of preoperative CT (Light Speed VCT; GE Yokokawa Medical Inc. Hino, Tokyo, JAPAN) for navigation is about $10 \mathrm{mGy}$ in the region between T1 
and L5. Intraoperative fluoroscopy for one minute results in an entrance surface dose of about $20 \mathrm{mGy}$, which is twice that of preoperative CT. Postoperative CT (SIEMENS SOMATOM Sensation 16; SIEMENS Asahi Meditec Inc. Shinagawa Tokyo JAPAN) had an entrance surface dose of about $23 \mathrm{mGy}$ in the region between T1 and L5. Though CT imaging for two sets results in a higher entrance surface dose, the dose is acceptable if CT imaging is limited to that for navigation.

The reported surgical time was as follows: the mean surgical time of segmental pedicle screw fixation by free had technique was reported to be 186 minutes and 341 minutes by Suk et $a l^{17}$ and Kim et $a l^{34}$, respectively. The mean surgical time with navigation system was reported to be 443 minutes by Sakai et al ${ }^{32}$. The reported intraoperative bleeding was as follows: the mean intraoperative bleeding with conventional methods for screw insertion was reported to be from $640 \mathrm{~mL}$ to $1241 \mathrm{~mL}^{17,34-37}$. The mean intraoperative bleeding with a navigation system was reported to be $1013 \mathrm{~mL}$ and $1100 \mathrm{~mL}$ by Sakai et al $^{32}$ and Rajasekaran et al ${ }^{30}$. In our study, the mean surgical time and mean intraoperative bleeding in multilevel registration using the navigation system was 310 minutes and $1138 \mathrm{~mL}$, respectively, and it was sufficiently less invasive.

This study had limitations in that it was retrospective and the number of patients was small. Additionally, we did not provide a comparative series of either the patients operated without a navigational device, or operated with registration at every level. However, most patients showed satisfactory results using the multilevel registration method of the CT-based navigation system. This technique may decrease operative time without compromising accuracy of pedicle screw placement.

\section{CONCLUSION}

Multilevel registration may decrease operative time without compromising accuracy of pedicle screw placement afforded by this technique in the setting of adolescent idiopathic scoliosis.

\section{ACKNOWLEDGMENT}

I am deeply grateful to Shigetaka Iimori, R.T. (radiology technologist), and Hiroshi Hirano, R.T., who measured entrance surface doses of CT and intraoperative fluoroscopy. 


\section{FIGURE LEGENDS}

Figure 1: Three consecutive vertebrae between T3 and T5 were registered together. Nine points were employed and mean registration error after point merge was as low as $1.1 \mathrm{~mm}$.

Figure 2: A 14-years-old girl received computer-assisted skip pedicle screw instrumentation using multilevel registration. The thoracic curve was corrected from 53 degrees to 10 degrees by Cobb angle. The correction rate was $81 \%$.

Figure 3: All pedicle screws showed grade 0.

Figure 4: Of the 265 pedicle screws, $244(92.1 \%)$ were categorized as grade $0,17(6.4 \%)$ were grade $1,3(1.1 \%)$ were grade 2 , and $1(0.4 \%)$ was grade 3 . Only one grade 3 screw was seen, in a right $\mathrm{T} 5$ pedicle. 
Accuracy of Single-time, Multilevel Registration

\section{REFERENCES}

1. Schwarzenbach O, Berlemann U, Jost B, et al. Accuracy of computer-assisted pedicle screw placement: an in vivo computed tomography analysis. Spine 1997;22:452-458.

2. Laine T, Schlenzka D, Makitalo K, et al. Improved accuracy of pedicle screw insertion with computer-assisted surgery: a prospective clinical Trial of 30 patients. Spine 1997;22:1254-1258. 3. Amiot LP, Lang K, Putzier M, et al. Comparative results between conventional and computerassisted pedicle screw installation in the thoracic, lumbar, and sacral spine. Spine 2000;25:606614.

4. Richter M, Amiot LP, Neller S, et al. Computer-assisted surgery in posterior instrumentation of the cervical spine: an in-vitro feasible study. Eur Spine J 2000;9(suppl 1):S65-S70.

5. Richter M, Cakir B, Schmidt R. Cervical pedicle screws: conventional versus computerassisted placement of cannulated screws. Spine 2005;30:2280-2287.

6. Kotani Y, Abumi K, Ito M, et al. Improved accuracy of computerassisted cervical pedicle screw insertion. J Neurosurg Spine 2003;99:257-263.

7. Seichi A, Takeshita K, Nakajima S, et al. Revision cervical spine surgery using transarticular or pedicle screws under a computer-assisted image-guidance system. J Orthop Sci 2005;10: 385-390.

8. Takahashi J, Shono Y, Nakamura I, et al. Computer-assisted screw insertion for cervical disorders in rheumatoid arthritis. Eur Spine J 2007; 16: 485-94.

9. Kalfas IH, Kormos DW, Murphy MA, et al. Application of frameless stereotaxy to pedicle screw fixation of the spine. J Neurosurg 1995;83:641-647.

10. Foley KT, Simon DA, Rampersaud YR. Virtual fluoroscopy: computer-assisted fluoroscopic navigation. Spine 2001;26:347-351.

11. Laine T, Lund T, Ylikoski M, et al. Accuracy of pedicle screw insertion with and without computer assistance. A randomized controlled clinical study in 100 consecutive patients. Eur Spine J 2000;9:235-240.

12. Schlenzka D, Laine T, Lund T. Computer-assisted spine surgery. Eur Spine J 2000;9(suppl):S57-S64.

13. Ebmeler K, Giest K, Kalff R. Intraoperative computerized tomography for improved accuracy of spinal navigation in pedicle screw placement of the thoracic spine. Acta Neurochir Suppl. 2003; 85:105-113.

14. Slomczykowski M, Roberto M, Schneeberger P, et al. Radiation dose for pedicle screw insertion. Fluoroscopic method versus computer-assisted surgery. Spine 1999;24:975-983.

15. Suk SI, Lee CK, Min HJ, et al. Comparison of Cotrel-Dubousset pedicle screws and hooks in the treatment of idiopathic scoliosis. Int Orthop 1994;18:341-346.

16. Halm H, Niemeyer T, Link T, et al. Segmental pedicle screw instrumentation in idiopathic thoracolumbar and lumbar scoliosis. Eur Spine J 2000;9:191-197.

17. Suk SI, Lee CK, KimWJ, et al. Segmental pedicle screw fixation in the treatment of thoracic idiopathic scoliosis. Spine 1995;20:1399-1405.

18. Liljenqvist UR, Halm HF, Link TM. Pedicle screw instrumentation of the thoracic spine in idiopathic scoliosis. Spine 1997;22:2239-2245.

19. Heini P, Scholl E,Wyler D, et al. Fatal cardiac tamponade associated with posterior spinal instrumentation. Spine 1998;23:2226-2230.

20. Papin P, Aebi M. Unusual presentation of spinal cord compression related to misplaced pedicle screws in thoracic scoliosis. Eur Spine J 1999;8:156-160. 
21. Liljenqvist UR, Link TM, Halm HF. Morphometric analysis of thoracic and lumbar vertebrae in idiopathic scoliosis. Spine 2000;25:1247-1253.

22. Holly LT, Foley KT. Intraoperative spinal navigation. Spine 2003;28:S54-61.

23. Papadopoulos EC, Girardi FP, Same A, et al. Accuracy of single-time, multilevel registration in image-guided spinal surgery. Spine J 2005;5:263-8.

24. Lenke LG, Betz RR, Harms J, et al. Adolescent idiopathic scoliosis: a new classification to determine extent of spinal arthrodesis. J Bone Joint Surg Am 2001;83:1169-81.

25. Lee SM, Suk SI, Chung ER. Direct vertebral rotation: A new technique of three-dimential deformity correction with segmental pedicle screw fixation in adolescent idiopathic scoliosis. Spine 2004; 29: 343-9.

26. Kadoury S, Cheriet F, Beausejour M, et al. A three-dimential retrospective analysis of the evolution of spinal instrumentation for the correction of adolescent idiopathic scoliosis. Eur Spine J 2009; 18: 23-37.

27. Rao G, Brofke DS, Rondina M, et al. Inter- and intraobserver reliability of computed tomography in assessment of thoracic pedicle screw placement. Spine 2003;28:2527-2530.

28. Suk SI, Lee SM, Chung ER, et al. Selective thoracic fusion with segmental pedicle screw fixation in the treatment of thoracic idiopathic scoliosis: more than 5-year follow-up. Spine 2005;30:1602-9,

29. Kim YJ, Lenke LG, Bridwell KH, et al. Free hand pedicle screw placement in the thoracic spine: is it safe? Spine 2004;29:333-42.

30. Rajasekaran S, Vidyadhara S, Ramesh P, et al. Randomized clinical study to compare the accuracy of navigated and non-navigated thoracic pedicle screws in deformity correction surgeries. Spine 2007;32:E56-64.

31. Kotani Y, Abumi K, Ito M, et al. Accuracy analysis of pedicle screw placement in posterior scoliosis surgery: comparison between conventional fluoroscopic and computer-assisted technique. Spine 2007;32:1543-50.

32. Sakai Y, Matsuyama Y, Nakamura H, et al. Segmental pedicle screwing for idiopathic scoliosis using computer-assisted surgery. J Spinal Disord Tech 2008;21:181-186.

33. Kuklo TR, Lenke LG, O'Brien MF, et al. Accuracy and efficacy of thoracic pedicle screws in curves more than 90 degrees. Spine 2005;30:222-226.

34. Kim YJ, Lenke LG, Cho SK, et al. Comparative analysis of pedicle screw versus hook instrumentation in posterior spinal fusion of adolescent idiopathic scoliosis. Spine 2004;29:20408.

35. Liljenqvist U, Lepsien U, Hackenberg L, et al. Comparative analysis of pedicle screw and hook instrumentation in posterior correction and fusion of idiopathic thoracic scoliosis. Eur Spine J 2002; 11: 336-43.

36. Chen I, Kim Y, Gupta MC, et al. Apical sublaminar wires versus pedicle screws - which provides better results for surgical correction of adolescent idiopathic scoliosis? Spine 2005; 30: 2104-12.

37. Kim YJ, Lenke LG, Kim J, et al. Comparative analysis of pedicle screw versus hybrid instrumentation in posterior spinal fusion of adolescent idiopathic scoliosis. Spine 2006; 31: 291 8. 


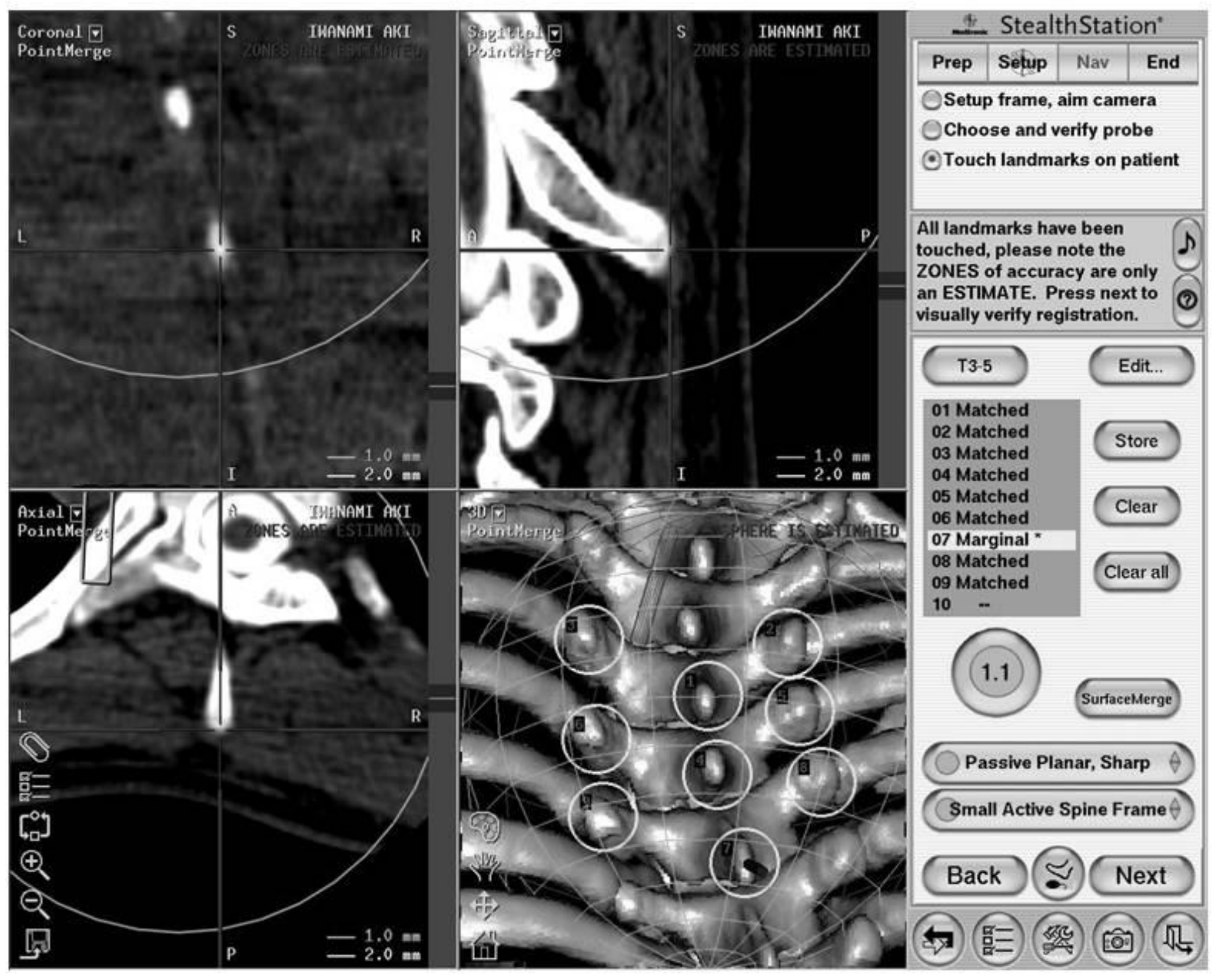

Figure 1 

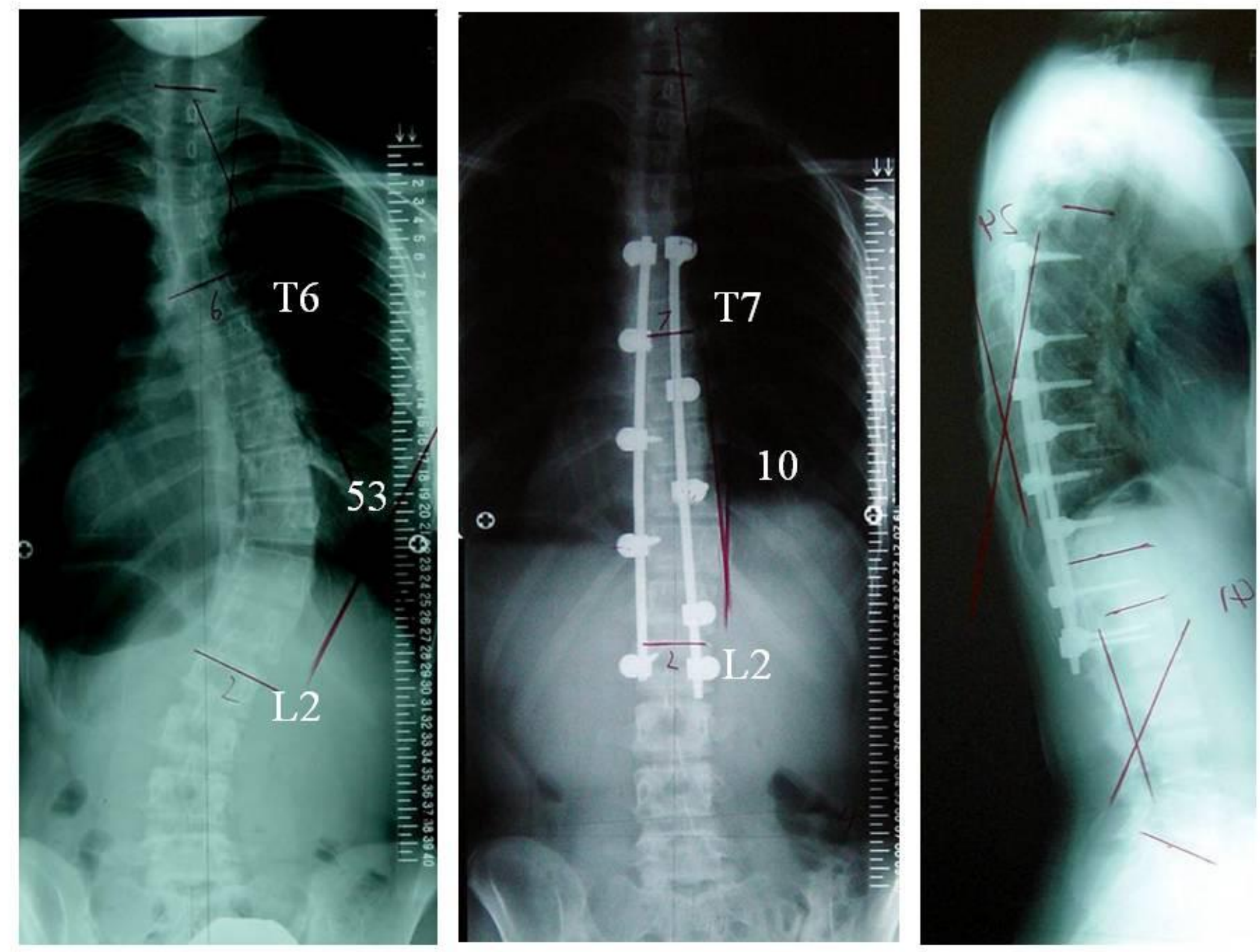

Figure 2 

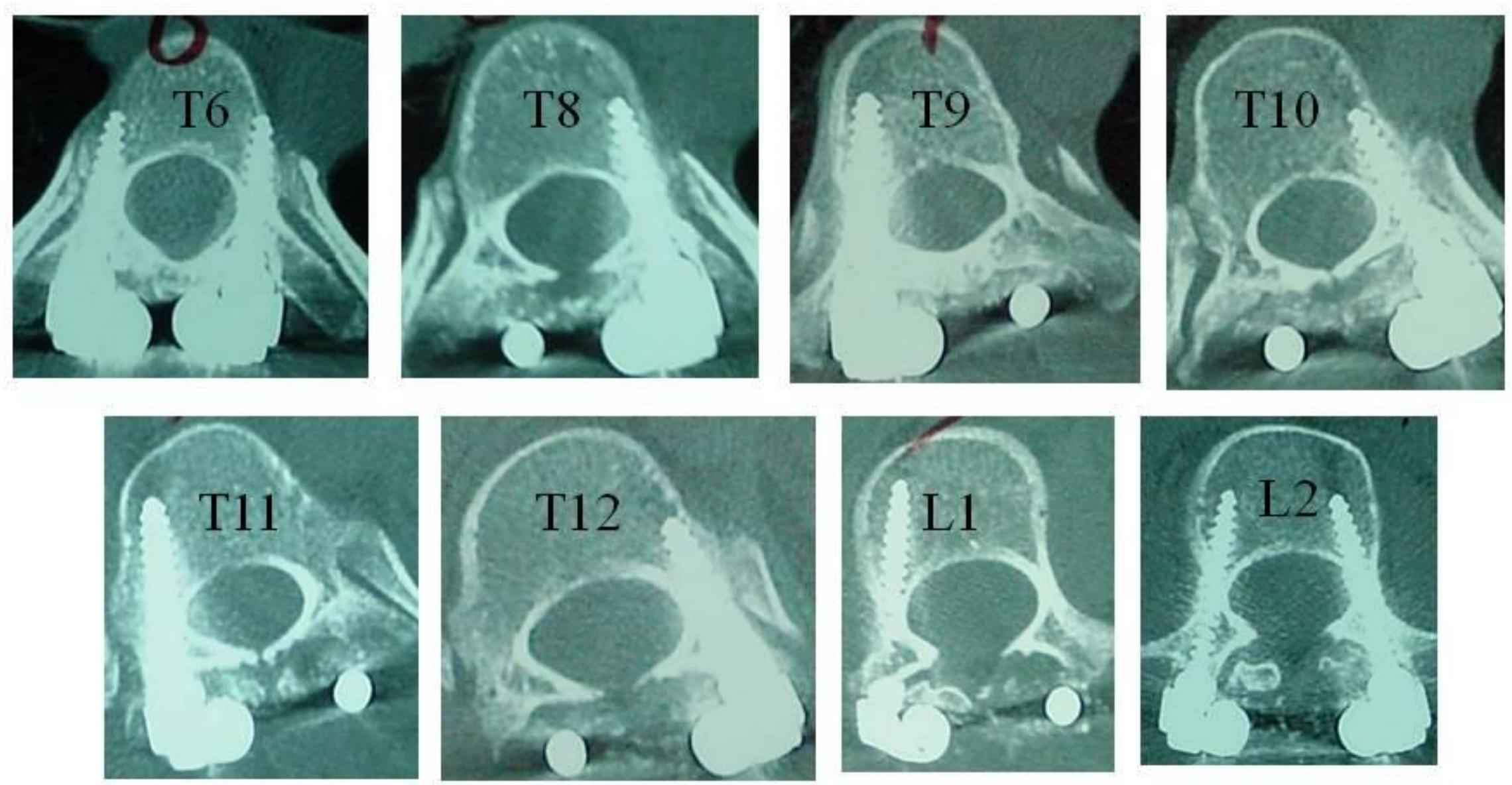

Figure 3 


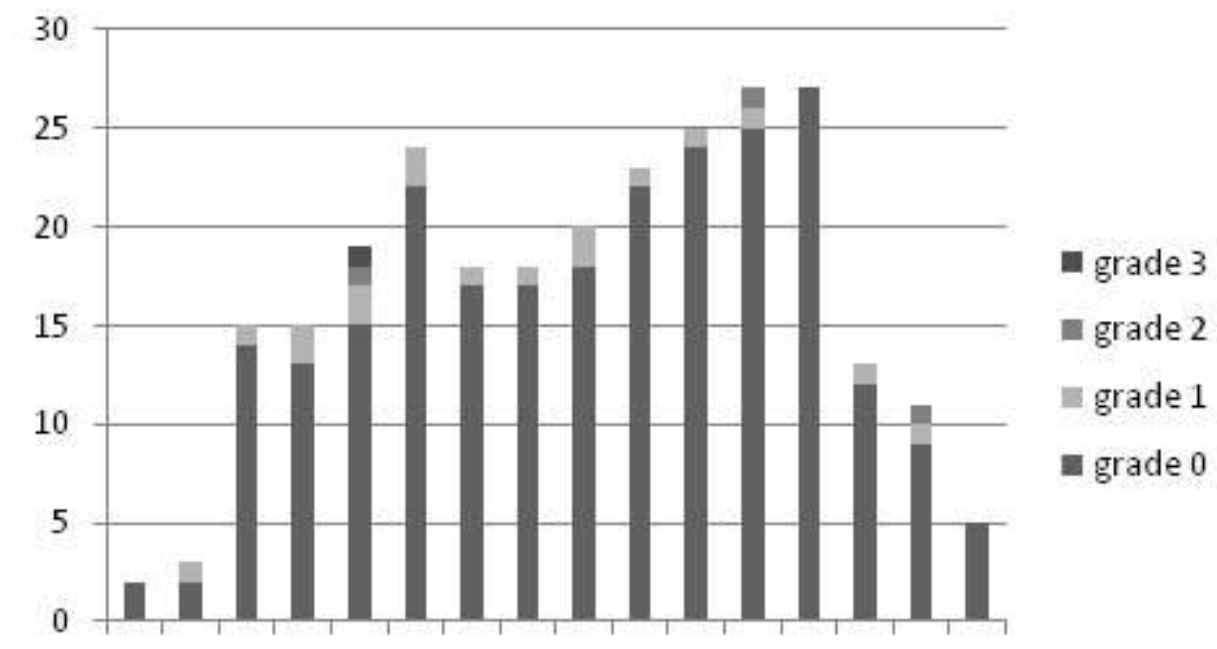

T1 T2 T3 T4 T5 T6 T7 T8 T9 T10T11T12 L1 L2 L3 L4

Figure 4 